\title{
A SYMMETRY PROPERTY FOR A CLASS OF RANDOM WALKS IN STATIONARY RANDOM ENVIRONMENTS ON $\mathbb{Z}$
}

\author{
JEAN-MARC DERRIEN $* * *$ AND \\ FRÉDÉRIQUE PLANTEVIN, * Université de Brest
}

\begin{abstract}
A correspondence formula between the laws of dual Markov chains on $\mathbb{Z}$ with two transition jumps is established. This formula contributes to the study of random walks in stationary random environments. Counterexamples with more than two jumps are exhibited.
\end{abstract}

Keywords: Markov chain; duality; random walk; stationary random environment; conductance and resistance

2010 Mathematics Subject Classification: Primary 60J10; 60K37

\section{Introduction}

If $\left(S_{n}\right)_{n \geq 0}$ refers to a random walk of law $v$ on the group $\mathbb{Z}$ of integers and if $\left(S_{n}^{*}\right)_{n \geq 0}$ is the random walk whose law $\stackrel{v}{v}$ is the inverse distribution of $v$, we obviously have, for any nonnegative integer $n$ and any $x$ in $\mathbb{Z}$,

$$
\mathrm{P}\left[S_{n}^{*}=-x \mid S_{0}^{*}=0\right]=\mathrm{P}\left[S_{n}=x \mid S_{0}=0\right] .
$$

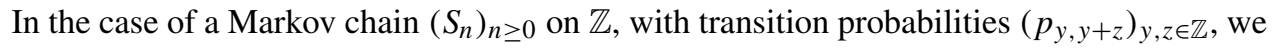
can consider a dual Markov chain $\left(S_{n}^{*}\right)_{n \geq 0}$ by taking as new transition probabilities

$$
p_{y, y+z}^{*}:=p_{y, y-z}, \quad y, z \in \mathbb{Z} \text {. }
$$

In [5] it was shown that the law of the return time at 0 for a birth-and-death Markov chain is easily expressed using the law of such a dual Markov chain. This kind of duality also appears in [3] and [4] for the study of random walks in stationary random environments on $\mathbb{Z}$.

For a Markov chain $\left(S_{n}\right)_{n \geq 0}$, equality (1) is obviously wrong in the general case as soon as $n \geq 2$.

The situation is slightly different in the case of random walks in stationary random environments. In what follows, we establish a quenched formula, the annealed version of which corresponds to (1) for random walks in stationary random environments on $\mathbb{Z}$ with the number of possible jumps at each site equal to exactly two. In Section 6 we give counterexamples when three jumps are allowed.

Received 12 July 2010; revision received 28 November 2011.

* Postal address: Département de Mathématiques, Université de Brest, UEB - 6, Avenue Victor Le Gorgeu, CS 93837,

29238 Brest Cedex 3, France.

** Email address: jean-marc.derrien@univ-brest.fr 


\section{Dual Markov chains, random walks in stationary random environments, and main results}

Let $a$ and $b$ be two elements of $\mathbb{Z}$ with $a>b$.

In the following, the Markov chains we consider are (time-homogeneous) Markov chains on $\mathbb{Z}$ with exactly two different jumps, $a$ and $b$, or possibly $-a$ and $-b$.

Let us denote by $\left(S_{n}\right)_{n \geq 0}$ such a Markov chain with jumps $a$ and $b$. The transition probabilities of $\left(S_{n}\right)_{n \geq 0}$ are simply given by the function

$$
\mathcal{P}: \mathbb{Z} \rightarrow(0,1), \quad y \mapsto p_{y},
$$

where $p_{y}:=p_{y, y+a}$. The probability $1-p_{y, y+a}$, which equals $p_{y, y+b}$, is also denoted by $q_{y}$.

In this paper, the dual Markov chain of $\left(S_{n}\right)_{n \geq 0}$ is the Markov chain $\left(S_{n}^{*}\right)_{n \geq 0}$ on $\mathbb{Z}$ with jumps $-a$ and $-b$ whose transition probabilities are given by

$$
p_{y, y-a}^{*}=p_{y, y+a} \quad \text { and } \quad p_{y, y-b}^{*}=p_{y, y+b} .
$$

Theorem 1 below shows a simple correspondence formula between the laws of $\left(S_{n}\right)_{n \geq 0}$ and $\left(S_{n}^{*}\right)_{n \geq 0}$. Our main motivation in this paper is to apply this theorem to random walks in stationary random environments on $\mathbb{Z}$.

Theorem 1. With the above notation, for any nonnegative integers $n, n_{a}$, and $n_{b}$ with $n_{a}+n_{b}=$ $n$, we have, for $x=n_{a} a+n_{b} b$,

$$
\begin{aligned}
\mathrm{P}\left[S_{n}^{*}=-x \mid S_{0}^{*}=0\right]= & \mathrm{P}\left[S_{n}=a \mid S_{0}=-x+a\right] \\
& +\left(\mathrm{P}\left[S_{n}>a \mid S_{0}=-x+a\right]-\mathrm{P}\left[S_{n}>b \mid S_{0}=-x+b\right]\right)
\end{aligned}
$$

for $n_{a} \geq 1$, and

$$
\mathrm{P}\left[S_{n}^{*}=-x \mid S_{0}^{*}=0\right]=\mathrm{P}\left[S_{n}=b \mid S_{0}=-x+b\right]
$$

for $n_{a}=0(x=n b)$.

Remark. The last equality of Theorem 1 is easy to prove:

$$
\begin{aligned}
\mathrm{P}\left[S_{n}^{*}=-n b \mid S_{0}^{*}=0\right] & =p_{0,-b}^{*} p_{-b,-2 b}^{*} \cdots p_{-(n-1) b,-n b}^{*} \\
& =p_{0, b} p_{-b, 0} \cdots p_{-(n-1) b,-(n-2) b} \\
& =\mathrm{P}\left[S_{n}=b \mid S_{0}=-n b+b\right] .
\end{aligned}
$$

The equality corresponding to the case $n_{a}=n$ could be established in the same way.

In the next three sections, we consider $n_{a} \geq 1$, first with an example (Section 3 ) and then in the general case (Sections 4 and 5).

We are now interested in the context of random walks in stationary random environments. The transition probabilities of $\left(S_{n}\right)_{n \geq 0}$ (and, thus, also of $\left.\left(S_{n}^{*}\right)_{n \geq 0}\right)$ are then given by realizations of a stationary sequence of random variables.

More precisely, considering an invertible measure-preserving transformation $\theta: \Omega \rightarrow \Omega$ on a probability space $(\Omega, \mathcal{F}, \mu)$ (see [7] for instance), we introduce a measurable function $p: \Omega \rightarrow[0,1]$ and, for a fixed $\omega$ in $\Omega$, we define the transition probabilities of $\left(S_{n}\right)_{n \geq 0}$ by

$$
p_{y}(\omega):=p\left(\theta^{y} \omega\right), \quad y \in \mathbb{Z} .
$$


Thus, for each $\omega$, we now have a probability $\mathrm{P}=\mathrm{P}^{\omega}$ that depends on $\omega$; it is the quenched law of the random walk in the environment given by $\omega$. The averaging probability with respect to the environments $\overline{\mathrm{P}}=\mathrm{P}^{\omega}(\cdot) \mu(\mathrm{d} \omega)$ is called the annealed law of the random walk in stationary random environments.

In this context, Theorem 1 can be rewritten as follows.

Theorem 2. (Theorem 1 reformulated.) With the above notation, for almost all $\omega$ and any nonnegative integers $n, n_{a}$, and $n_{b}$ with $n_{a}+n_{b}=n$, we have

$$
\begin{aligned}
\mathrm{P}^{\omega}\left[S_{n}^{*}=-x \mid S_{0}^{*}=0\right]= & \mathrm{P}^{\theta^{-x+a} \omega}\left[S_{n}=x \mid S_{0}=0\right] \\
& +\left(\mathrm{P}^{\theta^{-x+a} \omega}\left[S_{n}>x \mid S_{0}=0\right]-\mathrm{P}^{\theta^{-x+b} \omega}\left[S_{n}>x \mid S_{0}=0\right]\right)
\end{aligned}
$$

for $n_{a} \geq 1$, and

$$
\mathrm{P}^{\omega}\left[S_{n}^{*}=-x \mid S_{0}^{*}=0\right]=\mathrm{P}^{\theta^{-x+b} \omega}\left[S_{n}=-x \mid S_{0}=0\right]
$$

for $n_{a}=0$, where $x=n_{a} a+n_{b} b$.

As a corollary of Theorem 2, we obtain our second theorem which is concerned with the annealed case.

Theorem 3. In mean with respect to the environments, for any nonnegative integer $n$, the law of $S_{n}$ and the law of $-S_{n}^{*}$ are the same. In other words, for any $x$ in $\mathbb{Z}$,

$$
\overline{\mathrm{P}}\left[S_{n}^{*}=-x \mid S_{0}^{*}=0\right]=\overline{\mathrm{P}}\left[S_{n}=x \mid S_{0}=0\right] .
$$

We deduce in particular the following result.

Corollary 1. For any nonnegative integer n, we have the identity

$$
\overline{\mathrm{E}}\left(\left(S_{n}^{*}\right)^{2} \mid S_{0}^{*}=0\right)=\overline{\mathrm{E}}\left(S_{n}^{2} \mid S_{0}=0\right) .
$$

\subsection{Remark on the reversible case}

A conductance between two successive integers $y$ and $y+1$ is a positive number $c(y, y+1)$ and its inverse, $r(y, y+1)$, is the resistance between $y$ and $y+1$.

To a given family $(c(y, y+1))_{y \in \mathbb{Z}}$ of conductances, we can associate a nearest-neighbours Markov chain $\left(S_{n}\right)_{n \geq 0}$ on $\mathbb{Z}$ whose transition probabilities are proportional to the conductances. Thus, we have

$$
\mathrm{P}\left[S_{n+1}=y+1 \mid S_{n}=y\right]=\frac{c(y, y+1)}{\bar{c}(y)}
$$

and

$$
\mathrm{P}\left[S_{n+1}=y-1 \mid S_{n}=y\right]=\frac{c(y-1, y)}{\bar{c}(y)},
$$

where $\bar{c}(y):=c(y-1, y)+c(y, y+1)$.

When the conductances are obtained as realizations of a stationary sequence of positive random variables, we obtain a random walk in stationary random environments.

In the ergodic case, we can prove that, for almost all environments $\omega$ of conductances, the sequence $\left(\mathrm{E}^{\omega}\left(S_{n}^{2} \mid S_{0}=0\right) / n\right)_{n \geq 1}$ converges to the asymptotic variance

$$
\sigma^{2}=\frac{1}{\int c \mathrm{~d} \mu \int(1 / c) \mathrm{d} \mu},
$$


with the convention that $1 /+\infty=0$ (see [1], [2], and [6]). Therefore, we observe a symmetry between $c$ and $1 / c$ asymptotically in time.

For a fixed environment of conductances, if we replace conductances by resistances, we change the Markov chain governed by transition probabilities proportional to the conductances into a Markov chain whose transition probabilities are inversely proportional to the conductances. Thus, the Markov chain $\left(S_{n}\right)_{n \geq 0}$ becomes the dual chain $\left(S_{n}^{*}\right)_{n \geq 0}$ and we can apply Corollary 1 . This shows that the previous symmetry between $c$ and $1 / c$ appears in fact at any fixed time $n$ when averaging with respect to the environments.

\section{Initial observations: an example}

For this example, we consider a Markov chain $\left(S_{n}\right)_{n \geq 0}$ with jumps $a=1$ and $b=-1$ and we are interested in the probability

$$
\mathrm{P}^{\omega}\left[S_{n}=x \mid S_{0}=0\right]
$$

for $n=10$ and $x=2$.

We recall the following notation:

$$
p_{y}=p_{y, y+1} \quad \text { and } \quad q_{y}=p_{y, y-1}, \quad y \in \mathbb{Z} .
$$

First, let us consider the probability that $\left(S_{0}, S_{1}, S_{2}, \ldots, S_{10}\right)$ follows the path

$$
\Gamma:=(0,-1,0,1,2,3,4,3,2,3,2),
$$

starting at 0 and ending at 2; see Figure 1.

We have

$$
\mathrm{P}\left[\left(S_{0}, S_{1}, S_{2}, \ldots, S_{10}\right)=\Gamma\right]=q_{0} p_{-1} p_{0} p_{1} p_{2} p_{3} q_{4} q_{3} p_{2} q_{3} .
$$

Expanding this product with respect to each factor $q_{x}=1-p_{x}$, we obtain a sum with sixteen terms

$$
\begin{aligned}
\Sigma:= & 1 p_{-1} p_{0} p_{1} p_{2} p_{3} 11 p_{2} 1+1 p_{-1} p_{0} p_{1} p_{2} p_{3} 11 p_{2}\left(-p_{3}\right) \\
& +1 p_{-1} p_{0} p_{1} p_{2} p_{3} 1\left(-p_{3}\right) p_{2} 1+p_{-1} p_{0} p_{1} p_{2} p_{3} 1\left(-p_{3}\right) p_{2}\left(-p_{3}\right) \\
& +1 p_{-1} p_{0} p_{1} p_{2} p_{3}\left(-p_{4}\right) 1 p_{2} 1+1 p_{-1} p_{0} p_{1} p_{2} p_{3}\left(-p_{4}\right) 1 p_{2}\left(-p_{3}\right) \\
& +1 p_{-1} p_{0} p_{1} p_{2} p_{3}\left(-p_{4}\right)\left(-p_{3}\right) p_{2} 1 \\
& +1 p_{-1} p_{0} p_{1} p_{2} p_{3}\left(-p_{4}\right)\left(-p_{3}\right) p_{2}\left(-p_{3}\right) \\
& +\left(-p_{0}\right) p_{-1} p_{0} p_{1} p_{2} p_{3} 11 p_{2} 1+\left(-p_{0}\right) p_{-1} p_{0} p_{1} p_{2} p_{3} 11 p_{2}\left(-p_{3}\right) \\
& +\left(-p_{0}\right) p_{-1} p_{0} p_{1} p_{2} p_{3} 1\left(-p_{3}\right) p_{2} 1 \\
& +\left(-p_{0}\right) p_{-1} p_{0} p_{1} p_{2} p_{3} 1\left(-p_{3}\right) p_{2}\left(-p_{3}\right) \\
& +\left(-p_{0}\right) p_{-1} p_{0} p_{1} p_{2} p_{3}\left(-p_{4}\right) 1 p_{2} 1 \\
& +\left(-p_{0}\right) p_{-1} p_{0} p_{1} p_{2} p_{3}\left(-p_{4}\right) 1 p_{2}\left(-p_{3}\right) \\
& +\left(-p_{0}\right) p_{-1} p_{0} p_{1} p_{2} p_{3}\left(-p_{4}\right)\left(-p_{3}\right) p_{2} 1 \\
& +\left(-p_{0}\right) p_{-1} p_{0} p_{1} p_{2} p_{3}\left(-p_{4}\right)\left(-p_{3}\right) p_{2}\left(-p_{3}\right) .
\end{aligned}
$$

The main idea consists in transforming each term of $\Sigma$ in such a way to make appear the probabilities that the dual chain $\left(S_{n}^{*}\right)_{0 \leq n \leq 10}$ follows some paths. 


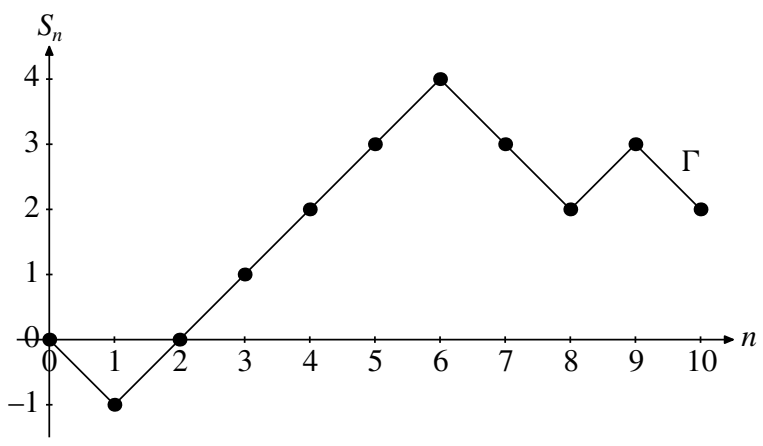

FIGURE 1: Representation of the path $\Gamma$.

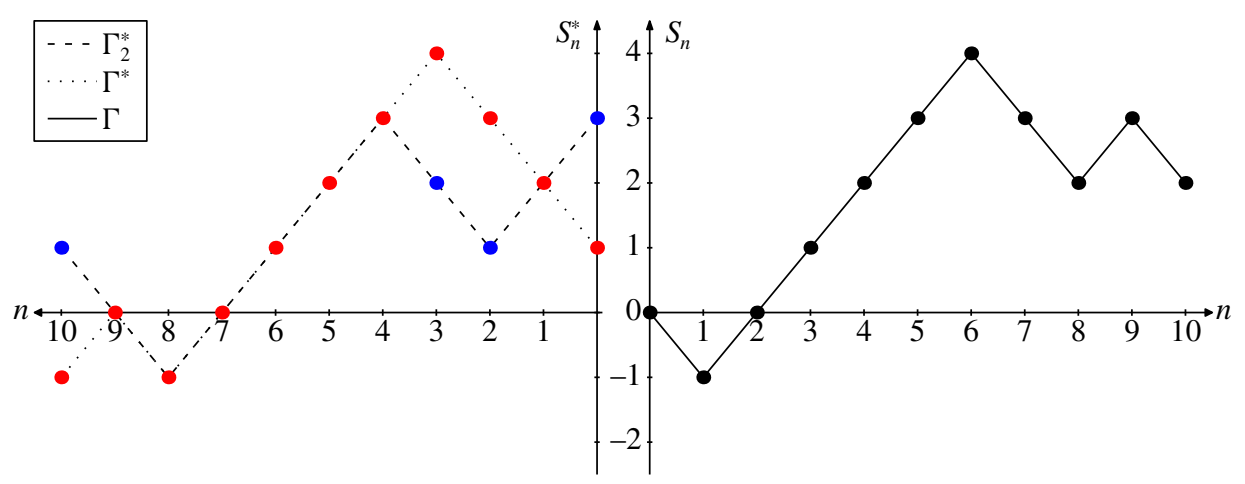

FiguRE 2: Representations of the paths $\Gamma, \Gamma^{*}$, and $\Gamma_{2}^{*}$.

Let us illustrate these transformations with the thirteenth term of $\Sigma$ :

$$
\left(-p_{0}\right) p_{-1} p_{0} p_{1} p_{2} p_{3}\left(-p_{4}\right) 1 p_{2} 1
$$

To take into account the order in which the factors of this product appear, we consider the 10-tuple

$$
\xi:=\left(-p_{0}, p_{-1}, p_{0}, p_{1}, p_{2}, p_{3},-p_{4}, 1, p_{2}, 1\right) .
$$

Then, we apply a mirror permutation, i.e.

$$
\left(1, p_{2}, 1,-p_{4}, p_{3}, p_{2}, p_{1}, p_{0}, p_{-1},-p_{0}\right),
$$

followed by a shift of the negative signs to the left (and cyclically), ignoring the terms equal to 1:

$$
\left(1,-p_{2}, 1, p_{4}, p_{3}, p_{2}, p_{1}, p_{0},-p_{-1}, p_{0}\right)=: \eta \text {. }
$$

Considering now the transition probabilities of the dual chain $\left(S_{n}^{*}\right)$, this 10-tuple $\eta$ reveals the path

$$
\Gamma^{*}:=(1,2,3,4,3,2,1,0,-1,0,-1)
$$

see Figure 2. Indeed, the product

$$
q_{1} q_{2} q_{3} p_{4} p_{3} p_{2} p_{1} p_{0} q_{-1} p_{0}=\mathrm{P}\left[\left(S_{0}^{*}, S_{1}^{*}, S_{2}^{*}, S_{3}^{*}, S_{4}^{*}, S_{5}^{*}, S_{6}^{*}, S_{7}^{*}, S_{8}^{*}, S_{9}^{*}, S_{10}^{*}\right)=\Gamma^{*}\right],
$$


expanded with respect to the quantities $q_{x}=1-p_{x}$, contains the term

$$
1\left(-p_{2}\right) 1 p_{4} p_{3} p_{2} p_{1} p_{0}\left(-p_{-1}\right) p_{0}
$$

the product of the coordinates of $\eta$.

With the same transformations as above, the first term of $\Sigma$, i.e.

$$
1 p_{-1} p_{0} p_{1} p_{2} p_{3} 11 p_{2} 1 \text {, }
$$

gives the 10-tuple $\left(1, p_{2}, 1,1, p_{3}, p_{2}, p_{1}, p_{0}, p_{-1}, 1\right)$, which is associated to the path $\Gamma_{1}^{*}:=$ $(1,2,1,2,3,2,1,0,-1,-2,-1)$.

For the second term of $\Sigma$,

$$
1 p_{-1} p_{0} p_{1} p_{2} p_{3} 11 p_{2}\left(-p_{3}\right),
$$

we obtain the 10-tuple $\left(p_{3}, p_{2}, 1,1, p_{3}, p_{2}, p_{1}, p_{0},-p_{-1}, 1\right)$, which is associated to the path $\Gamma_{2}^{*}:=(3,2,1,2,3,2,1,0,-1,0,1)$; see Figure 2 .

Continuing the same way reveals that

(i) each term of $\Sigma$ reveals a path $\Gamma_{i}^{*}(1 \leq i \leq 16)$;

(ii) each of the paths $\Gamma_{i}^{*}$ starts at 1 and ends at -1 , or starts at 3 and ends at 1 ;

(iii) none of the paths $\Gamma_{i}^{*}$ and $\Gamma_{j}^{*}$ such that $\Gamma_{i}^{*} \neq \Gamma_{j}^{*}$ are translations of each other;

(iv) considering all the terms of $\Sigma$ associated to the same path $\Gamma_{i}^{*}$ and summing the products of their transformed 10-tuple coordinates, we obtain only a part of the probability

$$
\mathrm{P}\left[\left(S_{0}^{*}, S_{1}^{*}, S_{2}^{*}, S_{3}^{*}, S_{4}^{*}, S_{5}^{*}, S_{6}^{*}, S_{7}^{*}, S_{8}^{*}, S_{9}^{*}, S_{10}^{*}\right)=\Gamma_{i}^{*}\right] .
$$

From (ii), it follows that a simple shift by ' -1 ' or ' -3 ' of the indexes of the $p_{y}$ makes the resultant paths $\Gamma_{i}^{*}$ all start at 0 and end at -2 .

Now, we claim that, when considering all the possible paths of length 10 starting at 0 and ending at 2 , we can reconstitute exactly the probability

$$
\mathrm{P}\left[S_{10}^{*}=-2 \mid S_{0}^{*}=0\right]
$$

by using the same procedure described above (see Corollary 2 below).

The following section makes precise and generalizes these remarks.

\section{Results needed for the proof of Theorem 1}

In this section we consider the general case of a Markov chain on $\mathbb{Z}$ with jumps $a$ and $b$ $(a>b)$ whose transition probabilities are given by the function

$$
\mathcal{P}: \mathbb{Z} \rightarrow(0,1), \quad y \mapsto p_{y},
$$

where $p_{y}=p_{y, y+a}$.

The sequence $\left(p_{y}\right)_{y \in \mathbb{Z}}$ will sometimes be regarded as a sequence of indeterminates, also denoted by $\mathcal{P}$. Distinguishing between the two uses is left to the reader.

Consider a positive integer $n$, and let $x=n_{a} a+n_{b} b$ be an $S_{n}$-reachable state, where $n_{a}$ and $n_{b}$ are nonnegative integers such that $n_{a}+n_{b}=n$.

Our aim is to establish a relationship between the law of $S_{n}^{*}$ and the probability

$$
\mathrm{P}\left[S_{n}=x \mid S_{0}=0\right]
$$

that the walk $\left(S_{n}\right)_{n \geq 0}$ reaches $x$ at time $n$ when it starts at 0 . 
We shall proceed in a combinatorial way and consider, as in the previous section, the probability for $S_{n}$ and $S_{n}^{*}$ to follow peculiar paths. To do this, we need to introduce further notation.

We denote by $\mathcal{C}_{0}^{(n)}$ the set of paths of length $n$ starting at 0 whose jumps are equal to $a$ or $b$ :

$$
\mathfrak{C}_{0}^{(n)}:=\left\{\Gamma=\left(\gamma_{0}, \gamma_{1}, \ldots, \gamma_{n}\right) \in \mathbb{Z}^{n+1} \mid \gamma_{0}=0, \gamma_{i+1}-\gamma_{i} \in\{a, b\}, i=0,1, \ldots, n-1\right\} .
$$

We denote by $\mathcal{C}_{0, s}^{(n)}$ for $s$ in $\mathbb{Z}$ the set of the paths in $\mathcal{C}_{0}^{(n)}$ which end at $s$ :

$$
\mathcal{C}_{0, s}^{(n)}:=\left\{\Gamma \in \mathcal{C}_{0}^{(n)} \mid \gamma_{n}=s\right\}
$$

We successively introduce

$$
\mathcal{E}(\mathcal{P}):=\bigcup_{y \in \mathbb{Z}}\left\{-p_{y}, 1, p_{y}\right\},
$$

and, for $\Gamma=\left(\gamma_{0}, \gamma_{1}, \ldots, \gamma_{n}\right)$ in $\mathcal{C}_{0}^{(n)}$,

$$
\begin{aligned}
\mathscr{D}_{\Gamma}(\mathcal{P}):=\left\{\xi=\left(\xi_{0}, \xi_{1}, \ldots, \xi_{n-1}\right) \in \mathcal{E}(\mathcal{P})^{n} \mid \xi_{i}=\right. & p_{\gamma_{i}} \text { if } \gamma_{i+1}=\gamma_{i}+a \\
& \text { or } \left.\xi_{i} \in\left\{1,-p_{\gamma_{i}}\right\} \text { if } \gamma_{i+1}=\gamma_{i}+b\right\} .
\end{aligned}
$$

Thus, we have

$$
\mathrm{P}\left[\left(S_{0}, S_{1}, \ldots, S_{n}\right)=\Gamma\right]=p_{\gamma_{0}, \gamma_{1}} p_{\gamma_{1}, \gamma_{2}} \cdots p_{\gamma_{n-1}, \gamma_{n}}=\sum_{\xi \in \mathcal{D}_{\Gamma}(\mathcal{P})} \prod_{i=0}^{n-1} \xi_{i}
$$

(this last identity is obtained by expanding the product $p_{\gamma_{0}, \gamma_{1}} p_{\gamma_{1}, \gamma_{2}} \cdots p_{\gamma_{n-1}, \gamma_{n}}$ with respect to each factor $\left.q_{y}=1-p_{y}\right)$.

Denoting by $\mathscr{D}(0, x, n, \mathcal{P})$ the disjoint union of the sets $\mathcal{D}_{\Gamma}(\mathcal{P})$ over $\Gamma$ in $\mathcal{C}_{0, x}^{(n)}$, we obtain the following proposition.

Proposition 1. With the above notation,

$$
\mathrm{P}\left[S_{n}=x \mid S_{0}=0\right]=\sum_{\xi \in \mathcal{D}(0, x, n, \mathcal{P})} \prod_{i=0}^{n-1} \xi_{i} .
$$

Similarly, we have

$$
\mathrm{P}\left[S_{n}^{*}=-x \mid S_{0}^{*}=0\right]=\sum_{\eta \in \mathcal{D}^{*}(0,-x, n, \mathcal{P})} \prod_{i=0}^{n-1} \eta_{i},
$$

where $\mathscr{D}^{*}(0,-x, n, \mathcal{P})$ is the set

$$
\begin{array}{r}
\bigcup_{\Gamma \in-\mathcal{C}_{0, x}^{(n)}}\left\{\eta=\left(\eta_{0}, \eta_{1}, \ldots, \eta_{n-1}\right) \in \mathcal{E}(\mathcal{P})^{n} \mid \eta_{i} \in\left\{1,-p_{\gamma_{i}}\right\} \text { if } \gamma_{i+1}=\gamma_{i}-b\right. \\
\text { or } \left.\eta_{i}=p_{\gamma_{i}} \text { if } \gamma_{i+1}=\gamma_{i}-a\right\} .
\end{array}
$$

Let us now suppose that $n_{a}$ is greater than or equal to 1 and introduce the notation

$$
i(\xi):=\max \left\{i \in\{0,1, \ldots, n-1\} \mid \xi_{i} \neq 1\right\} \text { for } \xi \in \mathscr{D}(0, x, n, \mathcal{P})
$$


and

$$
\mathscr{D}_{+}(0, x, n, \mathcal{P}):=\left\{\xi \in \mathscr{D}(0, x, n, \mathcal{P}) \mid \xi_{i(\xi)} \in\left\{p_{y} \mid y \in \mathbb{Z}\right\}\right\} .
$$

Remark. Given an element $\xi$ of $\mathcal{D}_{+}(0, x, n, \mathcal{P})$, then, by construction, the unique path $\Gamma$ in $\mathcal{C}_{0, x}^{(n)}$ associated to $\xi$ appears to make a jump equal to $a$ between the times $i(\xi)$ and $i(\xi)+1$, and continues to make jumps equal to $b$ thereafter until it reaches $x$. Hence, we have

$$
\gamma_{i(\xi)}=x-a-(n-i(\xi)-1) b .
$$

Furthermore, since $\Gamma$ has exactly $n_{b}$ jumps equal to $b$, we necessarily have

$$
i(\xi) \geq n-n_{b}-1=n_{a}-1 .
$$

The following proposition gives a probabilistic interpretation of the set $\mathscr{D}_{+}(0, x, n, \mathcal{P})$.

Proposition 2. For any positive integer $n$ and any $x=n_{a} a+n_{b} b$ with $n=n_{a}+n_{b}$ and $n_{a} \geq 1$,

$$
\mathrm{P}\left[S_{n} \geq x \mid S_{0}=0\right]=\sum_{\xi \in \mathcal{D}_{+}(0, x, n, \mathcal{P})} \prod_{i=0}^{n-1} \xi_{i} .
$$

Proof. Let $\Gamma=\left(\gamma_{0}, \gamma_{1}, \ldots, \gamma_{n}\right)$ be a path in $\mathcal{C}_{0}^{(n)}$ such that $\gamma_{n} \geq x$.

We begin by noting that, since the jumps of $\gamma$ are equal to $a$ or $b$, the function $i \mapsto-b i+\gamma_{i}$ is increasing with jumps equal to 0 or $a-b$. Its initial value $-b \cdot 0+\gamma_{0}=0$ is less than or equal to $\left(n_{a}-1\right)(a-b)=-b n+x-(a-b)\left(a>b\right.$ and $\left.n_{a} \geq 1\right)$ and its final value $-b n+\gamma_{n}$ is greater than or equal to $-b n+x$. Thus, there exists a unique instant $i_{0}$ in $\{0,1, \ldots, n-1\}$ such that

$$
-b i_{0}+\gamma_{i_{0}}=-b n+x-(a-b) \quad \text { and } \quad-b\left(i_{0}+1\right)+\gamma_{i_{0}+1}=-b n+x .
$$

Furthermore, $i_{0}$ must be greater than $n_{a}-1$.

It follows that

$$
\begin{aligned}
\mathrm{P}\left[S_{n}\right. & \left.\geq x \mid S_{0}=0\right] \\
& =\sum_{i_{0}=n_{a}-1}^{n-1} \mathrm{P}\left[i_{0}+S_{i_{0}}=-b n+x-(a-b),\left(i_{0}+1\right)+S_{i_{0}+1}=-b n+x \mid S_{0}=0\right] .
\end{aligned}
$$

Using the homogeneity in time of the transition probabilities of $\left(S_{n}\right)_{n \geq 0}$ and Proposition 1, we obtain, thanks to the remark before Proposition 2,

$$
\begin{aligned}
\mathrm{P}\left[S_{n} \geq x \mid S_{0}=0\right] & =\sum_{i_{0}=n_{a}-1}^{n-1} \mathrm{P}\left[S_{i_{0}}=-i_{0}-b n+x-(a-b) \mid S_{0}=0\right] p_{-i_{0}-b n+x-(a-b)} \\
& =\sum_{i_{0}=n_{a}-1}^{n-1}\left(\prod_{\xi \in \mathscr{D}\left(0,-i_{0}-b n+x-(a-b), i_{0}, \mathcal{P}\right)}^{i_{0}-1} \xi_{j=0}\right) p_{-i_{0}-b n+x-(a-b)} \\
& =\sum_{\xi \in \mathcal{D}_{+}(0, x, n, \mathcal{P})} \prod_{i=0}^{n-1} \xi_{i} .
\end{aligned}
$$

This completes the proof. 
For all $r$ in $\mathbb{Z}$, we now define a shift on $\mathcal{E}(\mathcal{P})$ by

$$
\theta^{r}\left(p_{y}\right):=p_{y+r}, \quad \theta^{r}(1):=1, \quad \theta^{r}\left(-p_{y}\right):=-p_{y+r}, \quad y \in \mathbb{Z},
$$

and we extend it on $\mathscr{E}(\mathcal{P})^{n}$ according to

$$
\theta^{r}(\xi):=\left(\theta^{r}\left(\xi_{0}\right), \theta^{r}\left(\xi_{1}\right), \ldots, \theta^{r}\left(\xi_{n-1}\right)\right), \quad \xi=\left(\xi_{0}, \xi_{1}, \ldots, \xi_{n-1}\right) \in \mathcal{E}(\mathcal{P})^{n} .
$$

We further define the three functions

$$
\varphi_{1}, \varphi_{2}, \varphi_{3}: \mathcal{E}(\mathcal{P})^{n} \rightarrow \mathcal{E}(\mathcal{P})^{n}
$$

by

$$
\begin{aligned}
& \varphi_{1}(\xi):= \begin{cases}\theta^{-x+a}(\xi) & \text { if } \xi \in D_{+}(0, x, n, \mathcal{P}), \\
\theta^{-x+b}(\xi) & \text { otherwise },\end{cases} \\
& \varphi_{2}\left(\left(\xi_{0}, \xi_{1}, \ldots, \xi_{n-1}\right)\right):=\left(\xi_{n-1}, \ldots, \xi_{1}, \xi_{0}\right),
\end{aligned}
$$

and, for all $i \in\{0,1, \ldots, n-1\}$,

$$
\left(\varphi_{3}(\xi)\right)_{i}:= \begin{cases}1 & \text { if } \xi_{i}=1, \\ \operatorname{sgn}\left(\xi_{\operatorname{succ}_{\xi}(i)}\right)\left|\xi_{i}\right| & \text { if } \xi_{i} \neq 1,\end{cases}
$$

where $\operatorname{succ}_{\xi}(i)$ denotes the index of the coordinate of $\xi$ which is not equal to 1 and which 'cyclically follows' the coordinate $\xi_{i}$. More precisely, if the set $\{j \in\{i+1, i+2, \ldots$, $\left.n-1\} \mid \xi_{j} \neq 1\right\}$ is not empty, we set

$$
\operatorname{succ}_{\xi}(i):=\min \left\{j \in\{i+1, i+2, \ldots, n-1\} \mid \xi_{j} \neq 1\right\}
$$

otherwise,

$$
\operatorname{succ}_{\xi}(i):=\min \left\{j \in\{0,1, \ldots, i\} \mid \xi_{j} \neq 1\right\} .
$$

Observe that $\varphi_{1}$ and $\varphi_{3}$ do not affect the coordinates of $\xi$ that are equal to 1 .

The composite mapping $\Phi:=\varphi_{3} \circ \varphi_{2} \circ \varphi_{1}$ allows us to write a first relation between the laws of $S_{n}$ and $S_{n}^{*}$.

Proposition 3. For any positive integer $n$ and any $x=n_{a} a+n_{b} b$ with $n=n_{a}+n_{b}$ and $n_{a} \geq 1$, the mapping $\Phi$ restricted to $\mathscr{D}(0, x, n, \mathcal{P})$ is one-to-one between the sets $\mathscr{D}(0, x, n, \mathcal{P})$ and $\mathcal{D}^{*}(0,-x, n, \mathcal{P})$ of Proposition 1.

Proof. Since the sets $\mathscr{D}(0, x, n, \mathcal{P})$ and $\mathscr{D}^{*}(0,-x, n, \mathcal{P})$ have the same cardinality, it suffices to prove that $\Phi: \mathscr{D}(0, x, n, \mathcal{P}) \rightarrow \mathcal{E}(\mathcal{P})^{n}$ is an injective mapping with values in $\mathscr{D}^{*}(0,-x, n, \mathcal{P})$.

The function $\Phi$ is injective because the functions

$$
\varphi_{1}: \mathcal{D}(0, x, n, \mathcal{P}) \rightarrow \mathcal{E}(\mathcal{P})^{n}, \quad \varphi_{2}: \mathcal{E}(\mathcal{P})^{n} \rightarrow \mathcal{E}(\mathcal{P})^{n}, \quad \text { and } \quad \varphi_{3}: \mathcal{E}(\mathcal{P})^{n} \rightarrow \mathcal{E}(\mathcal{P})^{n}
$$

are also injective. The injectivity of $\varphi_{1}: \mathscr{D}(0, x, n, \mathcal{P}) \rightarrow \mathscr{E}(\mathcal{P})^{n}$ is a consequence of the injectivity of the shifts $\theta^{r}, r \in \mathbb{Z}$, and the fact that the images by $\varphi_{1}$ of the sets $\mathscr{D}_{+}(0, x, n, \mathcal{P})$ and its complementary in $\mathscr{D}(0, x, n, \mathcal{P})$ are disjoint. The injectivity of $\varphi_{2}: \mathscr{E}(\mathcal{P})^{n} \rightarrow \mathscr{E}(\mathscr{P})^{n}$ is straightforward. The injectivity of $\varphi_{3}: \mathscr{E}(\mathscr{P})^{n} \rightarrow \mathscr{E}(\mathcal{P})^{n}$ is a consequence of the fact that, 
if we do not take into account the coordinates of $\xi$ in $\mathcal{E}(\mathcal{P})^{n}$ which are equal to 1 (and stay unchanged under the action of $\varphi_{3}$ ), the action of $\varphi_{3}$ on $\xi$ is a cyclic permutation of the signs of the remaining coordinates.

Let $\xi$ be an element of $\mathscr{D}(0, x, n, \mathcal{P})$. We prove the theorem by showing that $\Phi(\xi)$ belongs to the set $\mathscr{D}^{*}(0,-x, n, \mathcal{P})$. Set $\Phi(\xi)=\left(\eta_{0}, \eta_{1}, \ldots, \eta_{n-1}\right)$.

We have to only establish the existence of a path $\Gamma^{*}=\left(\gamma_{0}, \gamma_{1}, \ldots, \gamma_{n}\right)$ of $\mathcal{C}_{0}^{(n)}$ such that $\eta_{i} \in\left\{1,-p_{\gamma_{i}}\right\}$ when $\gamma_{i+1}=\gamma_{i}-b$ and $\eta_{i}=p_{\gamma_{i}}$ when $\gamma_{i+1}=\gamma_{i}-a$. (Note that such a path $\Gamma^{*}$ necessarily ends at $-x$ since $\Phi(\xi)$ contains the same number of coordinates belonging to $\left\{p_{y} \mid y \in \mathbb{Z}\right\}$ than $\xi$.)

Let $i_{0}$ be the minimum $i$ index such that $\eta_{i} \neq 1\left(i_{0}\right.$ exists because $\left.n_{a} \geq 1\right)$. Then existence of the path $\Gamma^{*}$ is a consequence of the following assertions:

(i) $\eta_{i_{0}} \in\left\{-p_{-i_{0} b}, p_{-i_{0} b}\right\}$;

(ii) for all $i$ in $\left\{i_{0}+1, i_{0}+2, \ldots, n-1\right\}$, if $\eta_{i} \in\left\{-p_{y}, p_{y}\right\}$ then either $\eta_{j}=-p_{y+(i-j) b}$ or $\eta_{j}=p_{y+(i-j-1) b+a}$, where $j$ is the greatest element of the set $\left\{i^{\prime} \mid i_{0} \leq i^{\prime} \leq\right.$ $i-1$ and $\left.\eta_{i^{\prime}} \neq 1\right\}$.

Let us start by proving (i). Because of the definition of $\Phi$ and since $\xi$ belongs to $\mathscr{D}(0, x, n, \mathcal{P})$, we have

$$
\xi_{n-i_{0}}=\xi_{n-i_{0}+1}=\cdots=\xi_{n-1}=1
$$

(this assertion is empty when $i_{0}=0$ ) and

$$
\xi_{n-1-i_{0}} \in\left\{-p_{x-\left(i_{0}+1\right) b}, p_{x-i_{0} b-a}\right\} .
$$

If $\xi_{n-1-i_{0}}=-p_{x-\left(i_{0}+1\right) b}$ then $\xi$ belongs to $\mathscr{D}(0, x, n, \mathcal{P}) \backslash \mathcal{D}_{+}(0, x, n, \mathcal{P})$ and we successively obtain

$$
\left(\varphi_{1}(\xi)\right)_{n-1-i_{0}}=-p_{-i_{0} b}, \quad\left(\varphi_{2} \circ \varphi_{1}(\xi)\right)_{i_{0}}=-p_{-i_{0} b},
$$

and

$$
\eta_{i_{0}}=\left(\varphi_{3} \circ \varphi_{2} \circ \varphi_{1}(\xi)\right)_{i_{0}} \in\left\{-p_{-i_{0} b}, p_{-i_{0} b}\right\} .
$$

In the same way, if $\xi_{n-1-i_{0}}=p_{x-i_{0} b-a}$ then $\xi$ is an element of $\mathscr{D}_{+}(0, x, n, \mathcal{P})$ and we have

$$
\left(\varphi_{1}(\xi)\right)_{n-1-i_{0}}=p_{-i_{0} b}, \quad\left(\varphi_{2} \circ \varphi_{1}(\xi)\right)_{i_{0}}=p_{-i_{0} b}
$$

and

$$
\eta_{i_{0}}=\left(\varphi_{3} \circ \varphi_{2} \circ \varphi_{1}(\xi)\right)_{i_{0}} \in\left\{-p_{-i_{0} b}, p_{-i_{0} b}\right\} .
$$

This completes the proof of (i).

To prove (ii), suppose that $i \in\left\{i_{0}+1, i_{0}+2, \ldots, n-1\right\}$ and that $\eta_{i} \in\left\{-p_{y}, p_{y}\right\}$. Denoting by $j$ the greatest element of the set $\left\{i^{\prime} \mid i_{0} \leq i^{\prime} \leq i-1\right.$ and $\left.\eta_{i^{\prime}} \neq 1\right\}$, we have, by the definition of $\Phi$,

$$
\xi_{n-1-i} \in\left\{-p_{y+r}, p_{y+r}\right\}, \quad \text { where } r=x-a \text { or } r=x-b
$$

and

$$
\xi_{n-i}=\xi_{n-i+1}=\cdots=\xi_{n-j-2}=1 .
$$

This implies that if $\xi_{n-1-i}=-p_{y+r}$ then

$$
\xi_{n-1-j} \in\left\{-p_{y+r+(i-j) b}, p_{y+r+(i-j) b}\right\},
$$


from which we deduce the identity

$$
\eta_{j}=-p_{y+(i-j) b}
$$

If $\xi_{n-1-i}=p_{y+r}$ then

$$
\xi_{n-1-j} \in\left\{-p_{y+r+(i-j-1) b+a}, p_{y+r+(i-j-1) b+a}\right\}
$$

and

$$
\eta_{j}=p_{y+(i-j-1) b+a} .
$$

This completes the proof of (ii) and, hence, the proposition.

Propositions 1 and 3 give at once the following result.

Corollary 2. For any positive integer $n$ and any $x=n_{a} a+n_{b} b$ with $n=n_{a}+n_{b}$ and $n_{a} \geq 1$,

$$
\mathrm{P}\left[S_{n}^{*}=-x \mid S_{0}^{*}=0\right]=\sum_{\xi \in \mathcal{D}(0, x, n, \mathcal{P})} \prod_{i=0}^{n-1}(\Phi(\xi))_{i} .
$$

\section{Proof of Theorem 1}

By the definition of $\Phi$, for any $\xi$ in $\mathscr{D}(0, x, n, \mathcal{P})$, we have

$$
\prod_{i=0}^{n-1}(\Phi(\xi))_{i}=\prod_{i=0}^{n-1} \theta^{r}\left(\xi_{i}\right)
$$

where $r=-x+a$ if $\xi$ is in $\mathscr{D}_{+}(0, x, n, \mathcal{P})$ and $r=-x+b$ otherwise.

From this, using Corollary 1 and Propositions 1 and 2, we derive

$$
\begin{aligned}
\mathrm{P}\left[S_{n}^{*}=-x \mid S_{0}^{*}=0\right]= & \sum_{\xi \in \mathcal{D}(0, x, n, \mathcal{P})} \prod_{i=0}^{n-1}(\Phi(\xi))_{i} \\
= & \theta^{-x+a}\left(\sum_{\xi \in \mathcal{D}_{+}(0, x, n, \mathcal{P})} \prod_{i=0}^{n-1} \xi_{i}\right) \\
& +\theta^{-x+b}\left(\sum_{\xi \in \mathcal{D}(0, x, n, \mathcal{P}) \backslash \mathcal{D}_{+}(0, x, n, \mathcal{P})} \prod_{i=0}^{n-1} \xi_{i}\right) \\
= & \theta^{-x+a}\left(\mathrm{P}\left[S_{n} \geq x \mid S_{0}=0\right]\right) \\
& +\theta^{-x+b}\left(\mathrm{P}\left[S_{n}=x \mid S_{0}=0\right]-\mathrm{P}\left[S_{n} \geq x \mid S_{0}=0\right]\right) \\
= & \theta^{-x+a}\left(\mathrm{P}\left[S_{n} \geq x \mid S_{0}=0\right]\right)-\theta^{-x+b}\left(\mathrm{P}\left[S_{n}>x \mid S_{0}=0\right]\right) \\
= & \theta^{-x+a}\left(\mathrm{P}\left[S_{n}=x \mid S_{0}=0\right]\right)+\theta^{-x+a}\left(\mathrm{P}\left[S_{n}>x \mid S_{0}=0\right]\right) \\
& -\theta^{-x+b}\left(\mathrm{P}\left[S_{n}>x \mid S_{0}=0\right]\right) .
\end{aligned}
$$

Here the probabilities have been regarded as polynomials in the indeterminates $p_{y}, y \in \mathbb{Z}$, and the shifts $\theta^{-x+a}$ and $\theta^{-x+b}$ have been extended to endomorphisms of the algebra $\mathbb{Z}\left[p_{y}\right.$; $y \in \mathbb{Z}$. 
Finally, for all $r, s$ in $\mathbb{Z}$, we have

$$
\theta^{r}\left(\mathrm{P}\left[S_{n}=s \mid S_{0}=0\right]\right)=\mathrm{P}\left[S_{n}=s+r \mid S_{0}=r\right] .
$$

This allows us to complete the proof.

\section{Counterexamples for random walks in stationary random environments with more than two jumps}

In this section, to any invertible ergodic measure-preserving transformation $\theta: \Omega \rightarrow \Omega$ on a nonatomic probability space $(\Omega, \mathcal{F}, \mu)$, we construct a random walk in stationary random environments with jumps $-1,1$, and 2 for which Theorem 2 does not hold.

By the hypotheses on $(\Omega, \mathcal{F}, \mu)$ and $\theta$, there exists an element $A$ of $\mathcal{F}$ with $\mu(A)>0$ and $\theta^{-3} A, \theta^{-2} A, A, \theta A$ pairwise disjoint sets (see [7]). Thus, we can consider

$$
\begin{aligned}
r(\omega) & :=\frac{1}{6}\left(\mathbf{1}_{A}(\omega)+\mathbf{1}_{\Omega}(\omega)\right), \\
p(\omega) & :=\frac{1}{6}\left(\mathbf{1}_{\theta^{-1} A}(\omega)+\mathbf{1}_{\Omega}(\omega)\right), \\
q(\omega) & :=1-p(\omega)-r(\omega),
\end{aligned}
$$

and characterize the law of the random walk in stationary random environments by

$$
\begin{aligned}
& \mathrm{P}^{\omega}\left[S_{n}=y+2 \mid S_{0}=y\right]=r\left(\theta^{y} \omega\right), \\
& \mathrm{P}^{\omega}\left[S_{n}=y+1 \mid S_{0}=y\right]=p\left(\theta^{y} \omega\right), \\
& \mathrm{P}^{\omega}\left[S_{n}=y-1 \mid S_{0}=y\right]=q\left(\theta^{y} \omega\right) .
\end{aligned}
$$

Then

$$
\begin{aligned}
\overline{\mathrm{P}}\left[S_{2}=\right. & \left.3 \mid S_{0}=0\right]-\overline{\mathrm{P}}\left[S_{2}^{*}=-3 \mid S_{0}^{*}=0\right] \\
= & \int\left(p(\omega) r(\theta \omega)+r(\omega) p\left(\theta^{2} \omega\right)-p(\omega) r\left(\theta^{-1} \omega\right)-r(\omega) p\left(\theta^{-2} \omega\right)\right) \mathrm{d} \mu(\omega) \\
= & \int r(\omega)\left(p\left(\theta^{-1} \omega\right)+p\left(\theta^{2} \omega\right)-p(\theta \omega)-p\left(\theta^{-2} \omega\right)\right) \mathrm{d} \mu(\omega) \\
= & \frac{1}{6} \int\left(p\left(\theta^{-1} \omega\right)+p\left(\theta^{2} \omega\right)-p(\theta \omega)-p\left(\theta^{-2} \omega\right)\right) \mathrm{d} \mu(\omega) \\
& +\frac{1}{6^{2}} \int \mathbf{1}_{A}(\omega)\left(\mathbf{1}_{A}(\omega)+\mathbf{1}_{\theta^{-3} A}(\omega)-\mathbf{1}_{\theta^{-2} A}(\omega)-\mathbf{1}_{\theta A}(\omega)\right) \mathrm{d} \mu(\omega) \\
= & \frac{1}{6^{2}} \mu(A) \\
> & 0,
\end{aligned}
$$

which yields the result.

Remark. We can similarly construct a random walk in stationary random environments with jumps $-1,0$, and 1 for which Theorem 2 does not hold.

\section{References}

[1] De Masi, A., Ferrari, P. A., Goldstein, S. and Wick, W. D. (1989). An invariance principle for reversible Markov processes. Applications to random motions in random environments. J. Statist. Phys. 55, 787-855.

[2] Depauw, J. and Derrien, J.-M. (2009). Variance limite d'une marche aléatoire réversible en milieu aléatoire sur ZZ C. R. Acad. Sci. Paris 347, 401-406. 
[3] Derriennic, Y. (1999). Random walks with jumps in random environments (examples of cycle and weight representations). In Probability Theory and Mathematical Statistics (Proc. 7th Internat. Vilnius Conf., August 1998), eds B. Grigelionis et al., pp. 199-212.

[4] DerrienNic, Y. (1999). Sur la récurrence des marches aléatoires unidimensionnelles en environnement aléatoire. C. R. Acad. Sci. Paris 329, 65-70.

[5] Dette, H., Fill, J. A., Pitman, J. and Studden, W. J. (1997). Wall and Siegmund duality relations for birth and death chains with reflecting barrier. J. Theoret. Prob. 10, 349-374.

[6] Kozlov, S. M. (1985). The method of averaging and walks in inhomogeneous environments. Russian Math. Surveys 40, 73-145.

[7] Petersen, K. (1983). Ergodic Theory (Camb. Stud. Adv. Math. 2). Cambridge University Press. 